\title{
Participação do Estado Contrátil e do Relaxamento Miocárdico na Disfunção Ventricular durante a Transição Hipertrofia-Falência Cardíaca
}

\author{
Antonio Carlos Cicogna, Kathleen G. Robinson, Chester H. Conrad, Robin Squire, \\ Marina P. Okoshi, Oscar H. L. Bing \\ Botucatu, SP e Boston, USA
}

Objetivo - Avaliar a participação do estado contrátil e do relaxamento miocárdico na disfunção do músculo cardíaco durante a transição hipertrofia-falência cardíaca em ratos espontaneamente hipertensos (SHR).

Métodos - Músculos papilares isolados do ventrículo esquerdo de SHR com insuficiência cardíaca (SHR-IC) $e$ sem falência cardíaca (SHR) e de ratos normotensos controle Wistar-Kyoto (WKY) foram estudados em contrações isométrica e isotônica, em solução de Krebs-Henseleit $\left(1,25 \mathrm{mMCa} \mathrm{Ca}^{2+}, 28^{\circ} \mathrm{C}\right)$.

Resultados - Os valores da tensão máxima desenvolvida $(T D)$ e da velocidade máxima de encurtamento $\left(V_{\text {máx }}\right)$ foram menores nos $S H R-I C$ e $S H R$, em relação aos $W K Y$ $(p<0,05)$. TD e $V_{\text {máx }}$ foram semelhantes nos SHR-IC e SHR $(p>0,05)$. A rigidez passiva do músculo aumentou significantemente nos SHR-IC ( $p<0,05$ vs WKY e SHR); esta variável não diferiu entre WKY e SHR ( $p>0,05)$.

Conclusão - Os dados obtidos mostram que a transição da fase de hipertrofia estável para insuficiência cardíaca nos ratos espontaneamente hipertensos está associada ao aumento da rigidez passiva do miocárdio e não à piora da função contrátil do músculo cardíaco.

Palavras-chave: hipertrofia, insuficiência cardíaca, função miocárdica

\section{Myocardial Function during the Transition from Compensated Left Ventricular Hyper- trophy to Failure}

Purpose - To investigate the participation of contractile state and relaxation in cardiac muscle dysfunction during the transition from stable hypertrophy to cardiac decompensation in aging spontaneously hypertensive rats (SHR).

Methods - Isolated left ventricular papillary muscle function was studied in SHR with heart failure (SHR-F), in age-matched SHR without evidence of heart failure (SHR$N F)$, and in nonhypertensive controls Wistar-Kyoto rats (WKY). Muscles were analised in isometric and isotonic contractions in Krebs-Henseleit solution with calcium concentration of $1.25 \mathrm{mM}$ at $28^{\circ} \mathrm{C}$.

Results - Papillary muscles from SHR-F and SHR-NF demonstrated decreased active tension development and shortening velocity relative to normotensive WKY $(p<0.05)$. SHR-F and SHR-NF did not differ. Compared with SHR-NF and WKY, muscle passive stiffness was increased in the failing SHR $(p<0.05$ versus WKY and SHR$N F)$. This parameter did not differ between SHR-NF and $W K Y(p>0.05)$.

Conclusion - These data suggest that the progression from stable hypertrophy to heart failure is associated with changes in the passive stiffness and is not related to depression of myocardial contractile function.

Key-words: hypertrophy, heart failure, myocardial function

Arq Bras Cardiol, volume 69 (n 6), 381-384, 1997

Faculdade de Medicina de Botucatu-UNESP e Veteran Administration Medical Center - Boston, USA. Apoio: FAPESP - Processo número 94/3203-7. Correspondência: Antonio Carlos Cicogna - Faculdade de Medicina de Botucatu - Depto de Clínica Médica - Rubião Júnior, S/N - 18618-000 - Botucatu, SP Recebido para publicação em 10/7/97

Aceito em 25/9/97
A hipertrofia cardíaca é um processo adaptativo que ocorre em resposta a uma série de estímulos fisiológicos e patológicos. A alteração na massa e na geometria da câmara permite ao ventrículo adaptar-se ao aumento do trabalho cardíaco. Entretanto, se o estímulo é persistente, podem surgir alterações na função miocárdica que levam à insuficiência cardíaca (IC) ${ }^{1}$. 
Os ratos espontaneamente hipertensos (SHR) foram desenvolvidos como um modelo genético de hipertensão arterial (HA), que é similar em muitos aspectos à HA essencial no homem ${ }^{2}$. Precocemente, os animais desenvolvem hipertrofia do ventrículo esquerdo (VE), que é responsável pela manutenção da função cardíaca normal, apesar da elevada pressão arterial sistêmica. Em idade mais avançada, podem ocorrer deterioração da função miocárdica e desenvolvimento de falência cardíaca ${ }^{3,4}$. A ocorrência da piora da disfunção ventricular e o aparecimento dos sinais clínicos de IC têm sido imputados à piora da contralidade e do relaxamento do músculo cardíaco ${ }^{1,5-9}$.

O resultado do presente trabalho, em discordância com os estudos mencionados ${ }^{1,5-9}$, mostra que a função contrátil do miocárdio de SHR manteve-se inalterada durante a transição de hipertrofia ventricular esquerda para IC. O aparecimento de falência cardíaca esteve associado somente ao aumento da rigidez passiva do miocárdio.

\section{Métodos}

Foram utilizados ratos machos, SHR e não hipertensos Wistar-Kyoto (WKY). A partir de 18 meses de idade, os animais passaram a ser observados diariamente a fim de se detectar a presença de taquipnéia. Quando determinados ratos apresentaram esse sintoma, considerou-se que estavam em IC e, portanto, em condições de serem estudados. A taquipnéia foi constatada somente em alguns SHR, não sendo notada nos WKY. No momento do sacrifício, procurou-se identificar outros sinais indicadores de IC, de acordo com achados prévios de Conrad e col ${ }^{1}$ : derrame pleuropericárdico, trombo no átrio esquerdo e hipertrofia ventricular direita (peso do ventrículo direito (VD)/peso corpóreo do rato maior que 0,80 ). Com base nas observações clínicas e patológicas foram constituídos três grupos de ratos com idades comparáveis: SHR com IC (SHR-IC, n=6), SHR não portador de IC (SHR, n=6) e WKY (n=5).

Após a decapitação, os corações foram removidos rapidamente e colocados em solução oxigenada de KrebsHenseleit ${ }^{10}$ a $28^{\circ} \mathrm{C}$. Os músculos papilares foram dissecados, presos em anéis de aço inoxidável e colocados verticalmente em uma câmara de acrílico, contendo $100 \mathrm{ml}$ de solução de Krebs-Henseleit, a $28^{\circ} \mathrm{C}$ e oxigenada com $95 \%$ de $\mathrm{O}_{2} \mathrm{e}$ $5 \%$ de $\mathrm{CO}_{2}(\mathrm{pH}=7,38)$. O músculo papilar mais fino e uniforme foi escolhido para o estudo. Os músculos foram estimulados 12 vezes por minuto por meio de eletrodos de platina, que liberavam pulsos de $5 \mathrm{~ms}$ de duração. A voltagem utilizada foi $10 \%$ maior que o valor mínimo necessário para provocar resposta mecânica máxima do músculo. A porção tendinosa superior do músculo, via anel e fio de aço inoxidável, foi conectada a um motor DC de baixa inércia (G100PD, General Scanning, Watertown, MA, USA). A extremidade inferior do músculo, via anel de aço, foi conectada a transdutor de força (DSC-3, Kistler-Morse, Redmond, WA, USA), imerso na solução. Um computador com interface digital-analógica permitiu controlar a tensão e o comprimento da preparação. Tensão e comprimento foram registrados à velocidade de $1 \mathrm{kHz}$ e armazenados em disquete para análise posterior.

Após período de 30min, em que os músculos contraíram isotonicamente contra leve carga (aproximadamente 0,4Kdina $/ \mathrm{mm}^{2}$ ), os mesmos foram colocados em contração isométrica. Nesta situação, foram estirados gradualmente até a tensão desenvolvida atingir o valor máximo. O comprimento diastólico da fibra muscular associado com a tensão máxima desenvolvida denomina-se $\mathrm{L}_{\text {máx }}$. Após atingir $\mathrm{L}_{\text {máx }}$, o músculo foi colocado durante $15 \mathrm{~min}$ em contração isotônica fisiológica contra pós-carga (pré-carga igual a $50 \%$ da pré-carga em $L_{\text {máx }}$ e pós-carga equivalente a $25 \%$ da tensão máxima desenvolvida). A seguir, novas determinações de $\mathrm{L}_{\text {máx }}$ foram realizadas. Após verificação da estabilidade de $\mathrm{L}_{\text {máx }}$, os músculos permaneceram em contração isométrica, sendo analisados os seguintes parâmetros: tensão máxima desenvolvida (TD,g/mm²), tensão de repouso (TR,g/mm²), velocidade máxima de variação temporal da TD (+dT/dt, g/mm²/s), velocidade máxima de variação temporal do relaxamento (-dT/dt, g/mm²/s), retardo eletromecânico (REM, ms), tempo para TD decrescer 50\% do seu valor máximo $\left(\mathrm{RT}_{1 / 2}, \mathrm{~ms}\right)$, e tempo para atingir o pico da tensão desenvolvida (TPT, ms). Em seguida, os músculos foram colocados em contração isotônica; a velocidade máxima de encurtamento $\left(\mathrm{V}_{\text {máx }}\right)$ foi registrada em $\mathrm{L}_{\text {máx }}$ com os músculos contraindo contra pós-carga próxima de zero.

Após determinação desses parâmetros basais, dois fios de seda (10-0 Dekmatel), distantes 1 a $2 \mathrm{~mm}$, foram passados ao redor dos músculos na região central e delicadamente presos aos mesmos. Após colocação dos dois marcadores, aferiu-se se a manobra afetou o desempenho dos músculos, determinando-se a tensão máxima desenvolvida. A aplicação de marcadores não afetou o desempenho da fibra cardíaca. A região do músculo entre as duas marcas foi utilizada para a determinação da rigidez central muscular. A medida da rigidez na zona central foi determinada com o auxílio de um sistema a laser para leitura do comprimento do segmento central do músculo ${ }^{11}$. A constante de rigidez do segmento central, $K_{c s}$ foi determinada pela relação $\log \left(s_{c s}\right)$ $=\mathrm{B}+\mathrm{K}_{\mathrm{cs}} \mathrm{E}_{\mathrm{cs}} ; \mathrm{s}_{\mathrm{cs}}$ é o estresse calculado a partir da tensão de repouso observada nos diferentes comprimentos do músculo, normalizada para as respectivas áreas de seção transversa; $\mathrm{E}_{\mathrm{cs}}$ é a deformação (strain) natural do segmento central $\left(\mathrm{E}_{\mathrm{cs}}=\ln \left[\mathrm{L}_{\mathrm{cs}} / \mathrm{L}_{0,1}\right]\right.$, onde $\mathrm{L}_{\mathrm{cs}}$ é o comprimento instantâneo do segmento central e $\mathrm{L}_{0,1}$ é o comprimento no estresse igual a $0,1 \mathrm{~g} / \mathrm{mm}^{2}$ ). Além da determinação de $\mathrm{K}_{\mathrm{cs}}$ foi também analisada a constante de rigidez de todo o músculo $\left(\mathrm{K}_{\mathrm{m}}\right)$.

Os parâmetros usados para caracterizar os músculos papilares foram: comprimento $(\mathrm{mm})$ e área seccional $\left(\mathrm{mm}^{2}\right)$. O comprimento in vitro, em $\mathrm{L}_{\text {máx }}$ foi obtido com catetômetro Gartner. A área seccional foi calculada, a partir de medidas do diâmetro do segmento central. Os pesos úmidos de VE, VD e átrios (A), normalizados para peso corpóreo do rato (PC), foram utilizados como índices de hipertrofia atrial e ventricular. A relação entre os pesos úmidos e secos dos ventrículos, átrios, fígado e pulmão foi utilizada como indicador da taxa de líquido presente em cada tecido corporal. 
Os valores obtidos são apresentados como média \pm desvio padrão. As comparações entre os grupos foram feitas por análise de variância para experimentos inteiramente casualizados, complementada pelo teste de Newman-Keuls. O nível de significância foi considerado a 5\%.

\section{Resultados}

Todos os animais do grupo SHR-IC apresentavam sinais e sintomas compatíveis com IC. Nos ratos dos grupos SHR e WKY não foi encontrado nenhum dos dados sugestivos de falência miocárdica, anteriormente descritos.

A idade e os parâmetros corporais dos animais encontram-se na tabela I. Não houve diferença de idade entre os animais. O peso corpóreo (PC) foi maior nos ratos WKY (WKY $>$ SHR $=$ SHR-IC, $\mathrm{p}<0,05)$. Opeso do VEdividido pelo peso corpóreo do rato (VE/PC) foi semelhante nos ratos SHReSHR-IC ( $p>0,05)$; entretanto, a relação VE/PC foi maior nos grupos SHR quando comparado aos WKY (SHR = SHR-IC $>$ WKY, $p<0,05)$. O peso do VD dividido pelo peso corpóreo dos ratos (VD/PC) foi maior nos ratos SHR-IC que nos SHR $(\mathrm{p}<0,05)$; não houve diferença entre os animais SHR e WKY $(p>0,05)$. A relação peso dos átrios/peso corpóreo dos ratos (A/PC) mostrou o mesmo comportamento da relação VD/PC. As relações entre o peso úmido e seco do VE(VEU/S) e do fígado (fígado U/S) foram maiores nos SHR-IC (SHR-IC $>$ SHR = WKY, $p<0,05)$. Não houve diferença entre os grupos de ratos quando comparam se as relações entre o peso úmido e seco de VD (VD U/S) e pulmão (pulmãoU/S).

Os dados mecânicos dos músculos papilares isolados são apresentados na tabela II. Não houve diferença significante entre as áreas seccionais dos três grupos de ratos. Tensão desenvolvida (TD) foi significantemente mais elevada nos ratos WKY do que nos SHR e SHR-IC ( $p<0,05)$. Não houve diferença entre os ratos dos grupos $\operatorname{SHR}(\mathrm{p}>0,05)$. $\mathrm{RT}_{1 / 2},+\mathrm{dT} / \mathrm{dt},-\mathrm{dT} / \mathrm{dte}_{\text {máx }}$ foram mais elevados nos WKY $(\mathrm{p}<0,05)$ quando comparados com os grupos SHR, que não se mostraram significantemente diferentes entre si (WKY $>$ SHR $=$ SHR-IC $)$. TPT foi mais elevado $(\mathrm{p}<0,05)$ nos SHR-

Tabela I - Idade e parâmetros corporais dos ratos estudados

\begin{tabular}{lccc|}
\hline & WKY $(\mathrm{n}=5)$ & SHR $(\mathrm{n}=6)$ & SHR-IC $(\mathrm{n}=6)$ \\
\hline Idade (meses) & $21,6 \pm 2,1$ & $19,2 \pm 1,0$ & $20,7 \pm 1,5$ \\
PC (g) & $668 \pm 84$ & $401 \pm 35^{*}$ & $407 \pm 21^{*}$ \\
VE/PC (mg/g) & $2,03 \pm 0,17$ & $3,72 \pm 0,22^{*}$ & $3,85 \pm 0,32^{*}$ \\
VD/PC (mg/g) & $0,50 \pm 0,11$ & $0,62 \pm 0,09$ & $1,12 \pm 0,09^{*+}$ \\
A/PC (mg/g) & $0,28 \pm 0,05$ & $0,40 \pm 0,07$ & $0,73 \pm 0,19^{*+}$ \\
VE (U/S) & $3,62 \pm 0,07$ & $3,74 \pm 0,10$ & $3,90 \pm 0,15^{*+}$ \\
VD (U/S) & $3,91 \pm 0,11)$ & $3,86 \pm 0,16$ & $3,98 \pm 0,10$ \\
Fígado (U/S) & $2,09 \pm 0,06$ & $2,13 \pm 0,10$ & $2,64 \pm 0,12^{*+}$ \\
Pulmão (U/S) & $4,12 \pm 0,23$ & $4,46 \pm 0,38$ & $4,28 \pm 0,15$ \\
\hline
\end{tabular}

Valores expressos em média \pm desvio padrão. WKY- ratos Wistar-Kyoto; SHRratos espontaneamente hipertensos; SHR-IC- SHR com insuficiência cardíaca; PC- peso corporal dos ratos; VE- peso do ventrículo esquerdo; VD- peso do ventrículo direito; A- peso dos átrios; U- peso úmido; S- peso seco; * $\mathrm{p}<0,05$ versus WKY; $+\mathrm{p}<0,05$ versus SHR.

\begin{tabular}{|lccc|}
\hline \multicolumn{4}{|c|}{ Tabela II - Parâmetros mecânicos obtidos em contrações } \\
isométricas e isotônicas
\end{tabular}

IC doque nos SHRe WKY (SHR-IC $>$ SHR $=$ WKY). $\mathrm{K}_{\mathrm{cs}} \mathrm{e} \mathrm{K}_{\mathrm{m}}$ foram significantemente maiores $(\mathrm{p}<0,05)$ nos SHR-IC, quando comparados aos ratos SHR e WKY; não houve diferença entre os animais SHRe WKY (SHR-IC $>$ SHR $=$ WKY $)$. TR e REM não diferiram entre os grupos ( $p>0,05)$.

\section{Discussão}

Nossos resultados mostraram que ratos com hipertrofia ventricular esquerda(SHR), isolada ou associada a IC, apresentaram redução dos indicadores da função contrátil $\left(\mathrm{TD},+\mathrm{dT} / \mathrm{dt}, \mathrm{V}_{\text {máx }}\right.$ ) e do relaxamento (-dT/dt, $\left.\mathrm{RT}_{1 / 2}\right)$ do músculo cardíaco, em relação aos animais com VE normal (WKY). Após o desenvolvimento do quadro clínico de falência cardíaca (grupo SHR-IC), os únicos parâmetros alterados foram os índices representativos da rigidez passiva do miocárdio, $\mathrm{K}_{\mathrm{cs}} \mathrm{e} \mathrm{K}_{\mathrm{m}}$. As variáveis indicativas do desempenho sistólico e do relaxamento não sofreram modificações significativas durante a transição hipertrofia-falência. Portanto, os dados mostraram que: 1) os músculos hipertrofiados dos SHR e SHR-IC apresentaram deterioração da função mecânica em relação às fibras cardíacas normais; 2) a disfunção ventricular nos SHR-IC esteve associada ao aumento da rigidez passiva do miocárdio e não à queda da função contrátil do músculo cardíaco.

Vários investigadores, utilizando diferentes tipos de preparações, estudaram as alterações fisiopatológicas ocorridas nos SHR durante a transição hipertrofia-IC 1,4,9. Conrad e col ${ }^{1,9}$ e Boluyt e col $^{4}$ verificaram deterioração da função contrátil e aumento da rigidez passiva dos músculos papilares isolados do VE no grupo SHR-IC. Bing e $\mathrm{col}^{8}$ constataram, em corações in vivo e em músculos ou corações isolados de SHR-IC, depressão do desempenho sistólico e piora da função diastólica, quando do apareci- 
mento dos sinais clínicos de IC. Os dados referidos contrastam, parcialmente, com os do presente trabalho, pois os diferentes autores encontraram disfunção sistólica e diastólica na transição hipertrofia-falência, enquanto que, nesta investigação, somente ocorreu piora da função diastólica.

Não encontramos explicações para esta divergência de resultados em relação ao comportamento sistólico dos músculos dos SHR-IC. Uma vez que fatores relacionados à metodologia poderiam ser afastados, já que os experimentos foram realizados no mesmo laboratório, uma provável explicação seria que, em nosso experimento, animais do grupo SHR foram sacrificados mais próximos do estado de IC do que os SHR utilizados em trabalhos anteriores. A favor desta hipótese está o fato de o comportamento mecânico sistólico dos SHR ser inferior ao dos WKY, o que contrasta com os dados prévios ${ }^{1,8,9}$, que mostram semelhança de função sistólica entre os animais WKY e SHR.

Os mecanismos que contribuem para o desenvolvimento de IC permanecem obscuros; várias hipóteses têm sido formuladas para explicar a anormalidade funcional existente no miocárdio hipertrofiado dos SHR. Há evidências que sugerem que perdas de proteínas contráteis, fibrose, alterações na composição das isoenzimas da miosina e do sistema acoplamento excitação-contração poderiam participar do processo de transição da hipertrofia para a falência cardíaca nos $\mathrm{SHR}^{4,7,12-14}$.

Boluyt e $\mathrm{col}^{4}$ verificaram aumento acentuado nos níveis de mRNA para colágeno e fibronectina no VE de SHR, durante o período de transição para IC. Os autores sugeriram que o aumento na expressão de genes específicos para a matriz extracelular poderia contribuir para fibrose, rigidez do tecido e deterioração da função ventricular. Conrad e col ${ }^{9}$ encontraram acentuada elevação do grau de fibrose intersticial, determinada histologicamente, em SHR-IC. Esses pesquisadores também sugerem que o aumento do tecido conjuntivo tem importante papel na transição hipertrofiafalência cardíaca. Na presente pesquisa, é possível inferir, baseado nos estudos citados ${ }^{4,9}$, que a elevação do grau de fibrose intersticial, acompanhando o quadro de IC, pudesse ter comprometido mais acentuadamente a rigidez passiva do miocárdio que a função sistólica. Concordando com esta hipótese, Cicogna e col ${ }^{15}$ mostraram que o aumento do tecido conjuntivo cardíaco pode acarretar disfunção diastólica mais precocemente que a sistólica.

O aumento da matriz extracelular durante o período de transição é atribuído à elevação de fatores circulantes e teciduais, como angiotensina II, aldosterona, catecolaminas e $\beta_{1}$ fator de transformação de crescimento (TGF- $\left.\beta_{1}\right)^{4,16}$. A angiotensina II tem importante papel na modulação da fibrose miocárdica. A administração de captopril a ratos SHR de 12 meses de idade previne o desenvolvimento de IC e de fibrose e o aumento de mRNAs para TGF- $\beta_{1}$ e para colágeno tipo III ${ }^{17}$.

Em conclusão, os dados deste trabalho mostram que o aparecimento da síndrome de IC em SHR está associado ao aumento da rigidez passiva do miocárdio e não à piora da função contrátil do músculo cardíaco. A disfunção diastólica pode ser decorrente de fibrose miocárdica provocada por fatores circulantes e teciduais, como angiotensina II, catecolaminas, aldosterona e TGF- $\beta_{1}$.

\section{Referências}

1. Conrad CH, Brooks WW, Robinson KG, Bing OHL - Impaired myocardial function in spontaneously hypertensive rats with heart failure. Am J Physiol 1991; 260: H136-H45.

2. Brooks WW, Bing OHL, Litwin SE, Conrad CH, Morgan JP-Effects of treppe and calcium on intracellular calcium and function in the failing heart from the spontaneously hypertensive rat. Hypertension 1994; 24: 347-56.

3. Pfeffer JM,Pfeffer MA, Fishbein MC, Frohlich ED-Cardiac function and morphology with aging in the spontaneously hypertensive rat. Am J Physiol 1979; 237: H461-H8.

4. Boluyt MO, Bing OHL, Lakatta EG - The ageing spontaneously hypertensive rat as a model of the transition from stable compensated hypertrophy to heart failure. Eur Heart J 1995; 16(suppl N): 19-30.

5. Pfeffer JM, Pfeffer MA, Fletcher P, Braunwald E- Alterations of cardiac performance in rats with established spontaneous hypertension. Am J Cardiol 1979; 44: 994-8.

6. Mirsky I, Pfeffer JM, Pfeffer MA, BraunwaldE-The contractile state as the major determinant in the evolution of left ventricular dysfunction in the spontaneously hypertensive rat. Circ Res 1983; 53: 767-78.

7. Boluyt MO, O'Neill L, Meredith AL et al - Alterations in cardiac gene expression during the transition from stable hypertrophy to heart failure. Marked upregulation of genes enconding extracellular matrix components. Circ Res 1994; 75: 23-32.

8. Bing OHL, Brooks WW, Robinson KG et al - The spontaneously hypertensive rat as a model of the transition from compensated left ventricular hypertrophy to failure. J Mol Cell Cardiol 1995; 27: 383-96.
9. Conrad CH, Brooks WW, Hayes JA, Sen S, Robinson KG, Bing OHL - Myocardial fibrosis and stiffness with hypertrophy and heart failure in the spontaneously hypertensive rat. Circulation 1995; 91: 161-70.

10. Krebs HA, Henseleit $K$ - Untersuchunger über die harnstoffbildung im tierkörper. Hoppe Seylers Z Physiol Chem 1932; 210: 33-66.

11. Wiegner AW, Bing OHL - Laser scanner measurement of central segment performance in isolated cardiac muscle preparations. Am J Physiol 1979; 237: H260H4.

12. Engelmann GL, Vitullo JC, Gerrity RG-Morphometric analysis of cardiac hypertrophy during development, maturation and senescence in spontaneously hypertensive rats. Circ Res 1987; 60: 487-94.

13. Perreault CL, Bing OHL, Brooks WW, Ransil BJ, Morgan JP-Differential effects of cardiac hypertrophy and failure on right versus left ventricular calcium activation. Circ Res 1990; 67: 707-12.

14. Bing OHL, Brooks WW, Conrad CH, Sen S, Perreault CL, Morgan JP-Intracellular calcium transients in myocardium from spontaneously hypertensive rats during the transition to heart failure. Circ Res 1991; 68: 1390-400.

15. Cicogna AC, Brooks WW, Hayes JA et al - Effect of chronic colchicine administration on the myocardium of the aging spontaneously hypertensive rat. Mol Cell Biochem 1997; 166: 45-54.

16. Boluyt MO, Bing OHL - The lonely failing heart: a case for ECM genes. Cardiovasc Res 1995; 30: 835-40.

17. Conrad CH, Boluyt MO, Brooks WW et al - Effect of captopril on TGF- $\beta_{1}$ gene expression, myocardial stiffness, and fibrosis in the spontaneously hypertensive rat. Circulation 1994; 90: I-261. 